\title{
Photohyperthermal therapy using liposomally formulated indocyanine green for feline nasal lymphoma: A case report
}

\author{
MASAMICHI YAMASHITA ${ }^{1}$, MAYUMI MAYAMA ${ }^{2}$, AKIKO SUGANAMI $^{3}$, KAZUO AZUMA ${ }^{1}$, TAKESHI TSUKA ${ }^{1}$, \\ NORIHIKO ITO ${ }^{1}$, TOMOHIRO IMAGAWA ${ }^{1}$, YUTAKA TAMURA $^{3}$ and YOSHIHARU OKAMOTO ${ }^{1}$ \\ ${ }^{1}$ Joint Department of Veterinary Clinical Medicine, Faculty of Agriculture, Tottori University, Tottori 680-8550; \\ ${ }^{2}$ Mayama Animal Hospital, Hokkaido 068-0028; ${ }^{3}$ Department of Bioinformatics, \\ Graduate School of Medicine, Chiba University, Chiba 260-8670, Japan
}

Received May 7, 2019; Accepted June 5, 2020

DOI: $10.3892 / \operatorname{mco} .2020 .2107$

\begin{abstract}
Our previous research has focused on the development of a novel cancer therapy by using photohyperthermal therapy (PHT) with indocyanine green (ICG) as an optical sensitizer. ICG-Lipo is a liposomally formulated ICG derivative in which ICG is tagged with an octadeca-alkyl chain to incorporate into liposome bilayers, and contains antitumor drugs such as carboplatin and paclitaxel within the inner membrane space. The present study reported a case of feline nasal lymphoma that was treated with combination therapy of PHT with ICG-Lipo. An antitumour effect was observed, and the patient entered remission. Complications from the radiation treatment included skin burns and bleeding from the irradiated hard palate. Serious side effects related to the drugs were not observed. This report suggested that PHT using ICG-Lipo enabled efficient and safe treatment of lymphoma, and that treatment with a liposomal drug delivery system was enhanced by PHT.
\end{abstract}

\section{Introduction}

Feline nasal lymphoma is the most common nasal tumour diagnosed in cats $(1,2)$. It is a relatively treatable tumour, with remission rates of $65-75 \%$ observed when various chemotherapy protocols are used (3). The median survival time (MST) of feline nasal lymphoma is 473 days with combined radiation therapy and chemotherapy, and 320 days with chemotherapy alone (4). Chemotherapy and radiation therapy are effective for the treatment of feline nasal lymphoma, and surgery is not required (4). Despite the effectiveness in treating feline nasal lymphoma, chemotherapy and radiation have

Correspondence to: Professor Yoshiharu Okamoto, Joint Department of Veterinary Clinical Medicine, Faculty of Agriculture, Tottori University, 4-101 Koyama-cho Minami, Tottori 680-8550, Japan

E-mail: yokamoto@muses.tottori-u.ac.jp

Key words: feline lymphoma, photohyperthermal therapy, liposome, drug delivery system, indocyanine green significant limitations; both have severe side effects, including bone marrow suppression and skin ulcers (5-8). Moreover, treatment is restricted by a repeated requirement for anaesthesia and high cost. The development of less burdensome and low-cost treatments would be beneficial in clinical settings.

Photohyperthermal therapy (PHT), combining photodynamic therapy (PDT) and photothermal therapy, has been investigated as safe and low-cost treatments for cancer (9-11). PHT is used in combination with near-infrared light (NIR) and a photosensitizer, such as indocyanine green (ICG) or aminolevulinic acid. The resulting activated oxygen has an anti-tumour effect $(10,11)$. ICG has a peak spectral absorption of $\sim 780 \mathrm{~nm}$ and peak fluorescence emission at $\sim 820 \mathrm{~nm}$. It induces heat and singlet oxygen formation in response to NIR light with a wavelength of $800 \mathrm{~nm}$ and is characterized by low general toxicity (10). The principal disadvantage of ICG is its rapid clearance from the body (plasmatic half-life of 2-4 min), limiting its accumulation within tumours (12). Several studies have reported the use of liposomally formulated ICG for optical imaging and cancer therapy, which improves its tumour-accumulating ability and stability (12-14). Liposomal drug delivery systems have enhanced permeability retention effects, which can increase the stability and accumulation within tumours of ICG $(15,16)$. Suganami et al $(13)$, designed and synthesised a novel NIR photoactivating probe, which is more hydrophobic compared with conventional ICG to promote liposome formation. Toyota et al (17), reported that a liposomally formulated ICG derivative (ICG-Lipo) yielded strong fluorescence images under an NIR-fluorescence imaging system. PDT using ICG-Lipo was reported to induce antitumour effects in vitro and in vivo $(18,19)$. The present study describes a case of feline lymphoma that was treated with the combination therapy of PHT with ICG-Lipo.

\section{Case report}

A 10-year-old male cat (weight, $4.1 \mathrm{~kg}$ ) presented with primary symptoms of sneezing, and nasal mucus and conjunctival injection in the right eye. The cat was initially diagnosed with an upper respiratory infection and prescribed an antibiotic. All the symptoms disappeared, with the exception of conjunctival hyperaemia. After 1 month, sneezing, nasal haemorrhage, protrusion of the right eye and facial swelling 


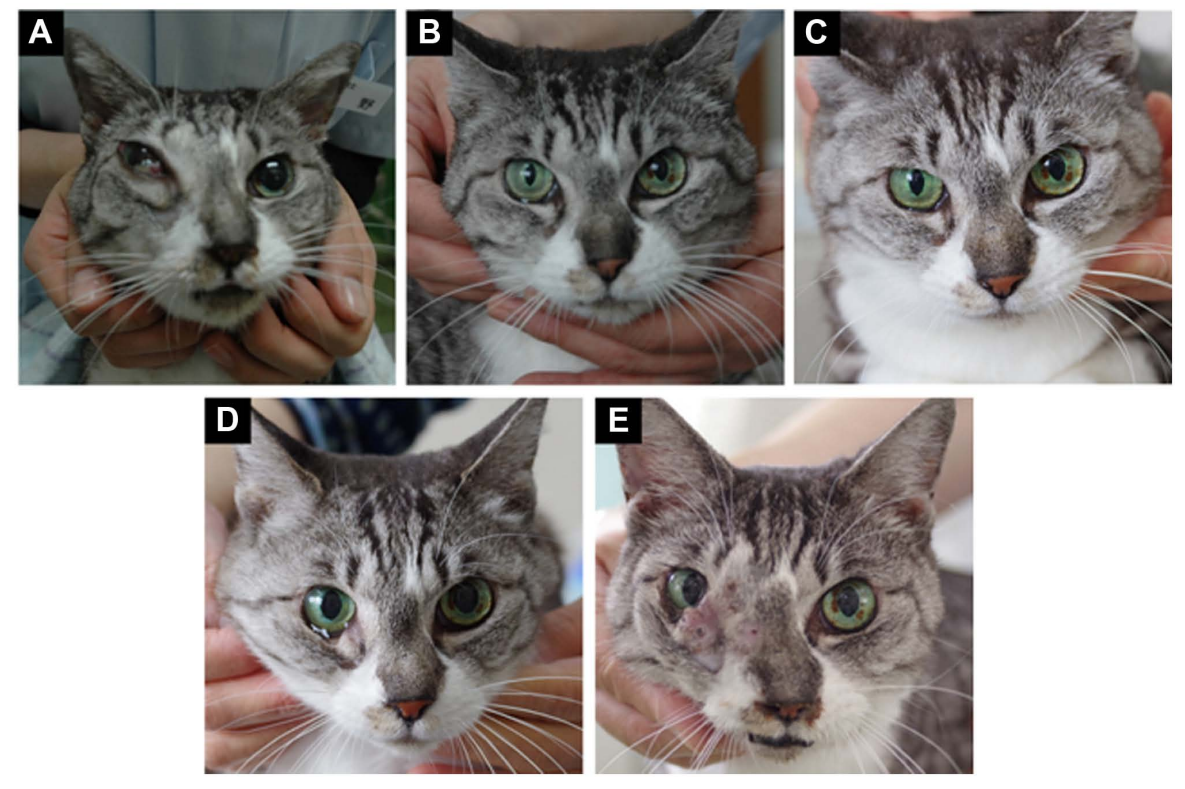

Figure 1. Gross appearance of the subject's face during the course of treatment. (A) Face is swollen, and the right third eyelid is observed on day 0. (B) Swelling disappeared on day 98 after the fifth course of PHT and (C) remained absent at 151 days. (D) Symptoms recurred on day 168 and (E) swelling of the face increased on day 201. PHT, photohyperthermal therapy.

were observed (Fig. 1A). Gingivitis was observed around the right upper premolar, which led to the diagnosis of a root abscess. Tooth extraction was performed with a routine course of post-operative antibiotics. However, the symptoms did not improve, and the facial swelling deteriorated. Cancer was suspected, and the owner was advised to seek treatment at a secondary hospital. The owner selected to have the cat treated at the primary hospital, where the combination therapy of PHT with ICG-Lipo was performed. This procedure was approved by the Organization for Research Initiative and Promotion in Tottori University (ethical approval no. H28-007).

ICG-Lipo, comprised of $2.25 \mathrm{mg}$ ICG-C18 (ICG derivative in which ICG is tagged with an octadeca-alkyl chain), $10 \mathrm{mg}$ carboplatin (Nichi-Iko Pharmaceutical Co., Ltd.) and $0.6 \mathrm{mg}$ paclitaxel (Bristol-Myers Squibb), was diluted with $50 \mathrm{ml}$ PBS $(\mathrm{pH}$ 7.4) at room temperature and administered intravenously at a rate of $50 \mathrm{ml} / \mathrm{h}$. Light irradiation was performed using a basic semiconductor laser (DVL-15; Asuka Medical, Inc.) five times a week for 2 weeks (Fig. 2). The total dose of light that the cat was subjected to per irradiation was $6,000 \mathrm{~J}$; light was irradiated at a dose rate of 5-10 W. Adjuvant therapy for the tumour was not administered. Fluid infusion was administered only when the cat exhibited a poor appetite. Five courses of combination therapy of PHT with ICG-Lipo were administered at 0, 25, 43, 60 and 74 days. Progressive improvement and deterioration of symptoms was observed between the first and fourth course of treatment. After the fifth course, facial swelling and nasal congestion showed dramatic improvement (Fig. 1B). Remission was confirmed and combination therapy was completed at the end of the fifth course. The tumour remained in remission for 151 days (Fig. 1C). The cat experienced skin burns and showed bleeding from the irradiated hard palate. These complications were treated by haemostasis under anaesthesia. No side effects of the antitumour drugs were observed, which was supported by complete blood counts between treatments. The symptoms reappeared after 168 days (95 days after the end of PHT; Fig. 1D)

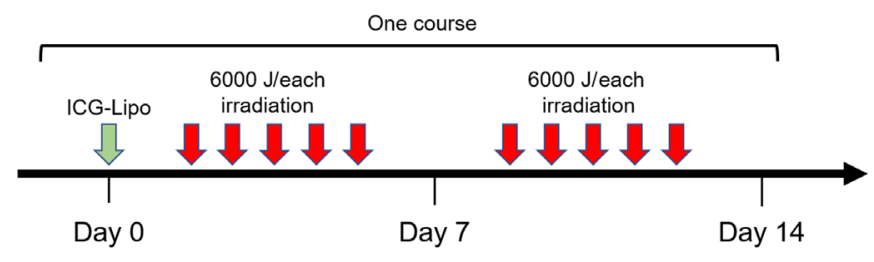

Figure 2. Treatment schedule of photohyperthermal therapy. A liposomal formulation of ICG was administered on day 0. Over 2 weeks, 10 courses of light irradiation were performed. The power of the light used for each session was $6,000 \mathrm{~J}$. ICG, indocyanine green.

and the facial swelling increased (Fig. 1E). Six additional courses of combination therapy were administered at 173, 202, 224, 239, 252, 273 and 359 days. The effects of the treatment were reduced compared with those observed during the first course of treatment. The cat was diagnosed with renal insufficiency on day 359 . The cat's general condition progressively worsened, and the cat was euthanised on day 401. The facial tumour was diagnosed as lymphoma and metastases were found in the kidney during necropsy. Immunohistochemical examination was not performed, as the owner did not desire it for economic reasons.

\section{Discussion}

The survival time (401 days) in the present case was longer than the MST (320 days) observed with chemotherapy alone (4). The increased survival time compared with the MST suggested that the combination therapy of PHT with ICG-Lipo is potentially effective for treating feline nasal lymphoma. The effects of the combination therapy were not observed until 2 months after the commencement of treatment, at the fifth course, when there was a dramatic decrease in the tumour. The effect of the treatment continued, and the remission phase lasted for $\sim 3$ months. It is proposed that continuous treatment is required for long-term remission. 
It was recently reported that the antitumour effect of the PHT/ICG-Lipo combination therapy was associated with an immunological mechanism (20). According to a report into using immunotherapy with programmed death (PD)-ligand 1 to treat a tumour, the tumour size temporarily increased and subsequently decreased (21). The tumour volume may have increased due to an inflammatory response. Alternatively, there may be a time-lag between the initiation of treatment and observation of the effect. In the present case, improvement and deterioration of the symptoms were observed throughout the course of treatment. The patient's response may have been a result of an increased immune response induced by PHT with ICG-Lipo.

However, significant treatment effects were not observed after relapse. The tumour cells may have become drug-resistant. If the antitumour effect was related to an immunological mechanism, the tumour may have expressed immune checkpoint molecules (such as PD-1 or cytotoxic T-lymphocyte associated protein 4). However, a biopsy could not be performed, thus it was not possible to compare molecular expressions between the primary and recurrent tumour. The immune effects of PHT with ICG-Lipo may be demonstrated via immunohistochemical examination for immune checkpoint molecules.

This is the first report of PHT with ICG-Lipo containing an antitumour drug. In this case, ICG-Lipo contained carboplatin and paclitaxel, and their dose was $\sim 10 \%$ of the standard administration protocol (22-24). Notable therapeutic effects were observed without the usual side effects of antitumour drugs. The encapsulation capacities of carboplatin and paclitaxel in liposomal pharmaceuticals were reported as 12 and $28 \%$, respectively (25). Antitumour effects were demonstrated in a previous study (25); however, the compound ratio of ICG-Lipo was not validated in this study. It is proposed that the compound ratio is similar to that utilised in the previous report (25), thus an antitumour effect was predicted.

In human medicine, nasal lymphoma is known as extranodal natural killer/T-cell lymphoma, nasal type (26). The lymphoma originates from either natural killer cells or $\gamma \delta$ T-cells, both of which express CD56 (26). Conversely, a B-cell phenotype comprises $40 \%$ of feline nasal lymphomas, and a T-cell phenotype comprises $47 \%$ (27). Moreover, the pathological features of human and feline nasal lymphomas are different. Thus, the findings from the present study may not contribute directly to human medicine. However, the treatment strategies for feline lymphoma are relatively similar to that for human lymphoma. It is proposed that PHT with ICG-Lipo has the potential to be effective in treating human lymphomas.

In conclusion, the present case report described the first treatment of nasal feline lymphoma using combination therapy of PHT with ICG-Lipo. The present case showed a satisfactory outcome compared with chemotherapy alone. PHT with ICG-Lipo is easy to perform and the side effects are less severe. However, a single case is insufficient to prove the exact treatment effects of PHT with ICG-Lipo. A clinical trial with a large study population is required to prove the efficacy of PHT with ICG-Lipo for treating nasal feline lymphoma.

\section{Acknowledgements}

Not applicable.

\section{Funding}

No funding was received.

\section{Availability of data and materials}

All data generated or analyzed during this study are included in this published article.

\section{Authors' contributions}

MY was involved in designing the treatment protocol of PHT with ICG-Lipo, evaluating the treatment effect and preparing the manuscript draft. MM oversaw all aspects of treatment and drafted a report on the case. AS and YT synthesised ICG-Lipo. KA and TT were involved in designing the fluid therapy and reviewing the manuscript. NI and TI were involved in analysing haematological data and reviewing the manuscript. YT and YO designed the treatment plan, and reviewed and edited the manuscript. All authors read and approved the final manuscript.

\section{Ethics approval and consent to participate}

The treatment protocol was approved by the Organization for Research Initiative and Promotion in Tottori University (ethical approval no. H28-007).

\section{Patient consent for publication}

The owner of the pet that was the subject of this case report provided written consent for the publication of the case report.

\section{Competing interests}

The author declare that they have no competing interests.

\section{References}

1. Henderson SM, Bradley K, Day MJ, Tasker S, Caney SM, Hotston Moore A and Gruffydd-Jones TJ: Investigation of nasal disease in the cat-a retrospective study of 77 cases. J Feline Med Surg 6: 245-257, 2004.

2. Mukaratirwa S, van der Linde-Sipman JS and Gruys E: Feline nasal and paranasal sinus tumours: Clinicopathological study, histomorphological description and diagnostic immunohistochemistry of 123 cases. J Feline Med Surg 3: 235-245, 2001.

3. Couto CG: Chapter 80: Lymphoma in the cat and dog. In: Nelson RW and Couto CG (eds.). Small animal internal medicine, 4th edition. St. Louis, Elsevier, pp1174-1186, 2009.

4. Haney SM, Beaver L, Turrel J, Clifford CA, Klein MK, Crawford S, Poulson JM and Azuma C: Survival analysis of 97 cats with nasal lymphoma: A multi-institutional retrospective study (1986-2006). J Vet Intern Med 23: 287-294, 2009.

5. Pinard CL, Mutsaers AJ, Mayer MN and Woods JP: Retrospective study and review of ocular radiation side effects following external-beam Cobalt-60 radiation therapy in 37 dogs and 12 cats. Can Vet J 53: 1301-1307, 2012.

6. Knapp DW, Richardson RC, Bonney PL and Hahn K: Cisplatin therapy in 41 dogs with malignant tumors. J Vet Intern Med 2 : 41-46, 1988.

7. Machado MC, da Costa-Neto JM, Portela RD, D'Assis MJMH, Martins-Filho OA, Barrouin-Melo SM, Borges NF, Silva FL and Estrela-Lima A: The effect of naltrexone as a carboplatin chemotherapy-associated drug on the immune response, quality of life and survival of dogs with mammary carcinoma. PLos One 13: $\mathrm{e} 0204830,2018$. 
8. Oun R, Moussa YE and Wheate NJ: The side effects of platinum-based chemotherapy drugs: A review for chemists. Dalton Trans 47: 6645-6653, 2018

9. O'Reilly S, Rowinsky E, Slichenmyer W, Donehower RC, Forastiere A, Ettinger D, Chen TL, Sartorius S, Bowling K, Smith J, et al: Phase I and pharmacologic studies of topotecan in patients with impaired hepatic function. J Natl Cancer Inst 88: 817-824, 1996

10. Radzi R, Osaki T, Tsuka T, Imagawa T, Minami S, Nakayama Y and Okamoto Y: Photodynamic hyperthermal therapy with indocyanine green (ICG) induces apoptosis and cell cycle arrest in B16F10 murine melanoma cells. J Vet Med Sci 74: 545-551, 2012.

11. Urbanska K, Romanowska-Dixon B, Matuszak Z, Oszajca J, Nowak-Sliwinska P and Stochel G: Indocyanine green as a prospective sensitizer for photodynamic therapy of melanomas. Acta Biochim Pol 49: 387-391, 2002.

12. Porcu EP, Salis A, Gavini E, Rassu G, Maestri M and Giunchedi P: Indocyanine green delivery systems for tumour detection and treatments. Biotechnol Adv 34: 768-789, 2016

13. Suganami A, Toyota T, Okazaki S, Saito K, Miyamoto K, Akutsu Y, Kawahira H, Aoki A, Muraki Y, Madono T, et al: Preparation and characterization of phospholipid-conjugated indocyanine green as a near-infrared probe. Bioorg Med Chem Lett 22: 7481-7485, 2012.

14. Xue X, Fang T, Yin L, Jiang J, He Y, Dai Y and Wang D Multistage delivery of CDs-DOX/ICG-loaded liposome for highly penetration and effective chemo-photothermal combination therapy. Drug Deliv 25: 1826-1839, 2018

15. Fang J, Nakamura $H$ and Maeda H: The EPR effect: Unique features of tumor blood vessels for drug delivery, factors involved, and limitations and augmentation of the effect. Adv Drug Deliv Rev 63: 136-151, 2011.

16. Matsumura $\mathrm{Y}$ and Maeda $\mathrm{H}$ : A new concept for macromolecular therapeutics in cancer chemotherapy: Mechanism of tumoritropic accumulation of proteins and the antitumor agent smancs. Cancer Res 46: 6387-6392, 1986.

17. Toyota T, Fujito H, Suganami A, Ouchi T, Ooishi A, Aoki A, Onoue K, Muraki Y, Madono T, Fujinami M, et al: Near-infrared-fluorescence imaging of lymph nodes by using liposomally formulated indocyanine green derivatives. Bioorg Med Chem 22: 721-727, 2014.

18. Suganami A, Iwadate Y, Shibata S, Yamashita M, Tanaka T, Shinozaki N, Aoki I, Saeki N, Shirasawa H, Okamoto Y and Tamura Y: Liposomally formulated phospholipid-conjugated indocyanine green for intra-operative brain tumor detection and resection. Int J Pharm 496: 401-406, 2015.
19. Maruyama T, Akutsu Y, Suganami A, Tamura Y, Fujito $\mathrm{H}$, Ouchi T, Akanuma N, Isozaki Y, Takeshita N, Hoshino I, et al: Treatment of near-infrared photodynamic therapy using a liposomally formulated indocyanine green derivative for squamous cell carcinoma. PLoS One 10: e0122849, 2015.

20. Shibata S, Shinozaki N, Suganami A, Ikegami S, Kinoshita Y, Hasegawa R, Kentaro H, Okamoto Y, Aoki I, Tamura Y and Iwadate Y: Photo-immune therapy with liposomally formulated phospholipid-conjugated indocyanine green induces specific antitumor responses with heat shock protein-70 expression in a glioblastoma model. Oncotarget 10: 175-183, 2019.

21. Brahmer JR, Tykodi SS, Chow LQ, Hwu WJ, Topalian SL, Hwu P, Drake CG, Camacho LH, Kauh J, Odunsi K, et al: Safety and activity of anti-PD-L1 antibody in patients with advanced cancer. N Engl J Med 366: 2455-2465, 2012.

22. Gustafson DL and Page RL: Cancer Chemotherapy. In: Withrow SJ, Vail DM and Page RL (eds.). Small animal clinical oncology, 5th edition. St. Louis, Elsevier, pp157-179, 2012

23. Kisseberth WC, Vail DM, Yaissle J, Jeglum KA, Couto CG, Ward H, Khanna C and Obradovich JE: Phase I clinical evaluation of carboplatin in tumor-bearing cats: A veterinary cooperative oncology group study. J Vet Intern Med 22: 83-88, 2008.

24. Poirier VJ, Hershey AE, Burgess KE, Phillips B, Turek MM, Forrest LJ, Beaver L and Vail DM: Efficacy and toxicity of paclitaxel (Taxol) for the treatment of canine malignant tumors. J Vet Intern Med 18: 219-222, 2004.

25. Zhang X, Liu Y, Kim YJ, Mac J, Zhuang R and Wang P: Co-delivery of carboplatin and paclitaxel via cross-linked multilamellar liposomes for ovarian cancer treatment. RSC Adv 7: 19685-19693, 2017.

26. Harabuchi Y, Takahara M, Kishibe $K$, Nagato $T$ and Kumai T: Extranodal natural Killer/T-cell lymphoma, nasal type: Basic science and clinical progress. Front Pediatr 7: 141, 2019.

27. Nagata K, Lamb M, Goldschmidt MH, Duda L and Walton RM: The usefulness of immunohistochemistry to differentiate between nasal carcinoma and lymphoma in cats: 140 Cases (1986-2000). Vet Comp Oncol 12: 52-57, 2014. 